\title{
Universiteit
}

Leiden

The Netherlands

\section{Spin diffusion with 13C selection and detection for the characterization of morphology in labeled polymer blends with MAS NMR}

Mulder, F.M.; Heinen, W.; Duin, M. van; Lugtenburg, J.; Groot, H.J.M. de

\section{Citation}

Mulder, F. M., Heinen, W., Duin, M. van, Lugtenburg, J., \& Groot, H. J. M. de. (1998). Spin diffusion with $13 \mathrm{C}$ selection and detection for the characterization of morphology in labeled polymer blends with MAS NMR. Journal Of The American Chemical Society, 120(49), 12891-12894. doi:10.1021/ja9809511

Version: $\quad$ Publisher's Version

License: $\quad$ Licensed under Article 25fa Copyright Act/Law (Amendment Taverne)

Downloaded from: https://hdl.handle.net/1887/3238650

Note: To cite this publication please use the final published version (if applicable). 


\title{
Spin Diffusion with ${ }^{13} \mathrm{C}$ Selection and Detection for the Characterization of Morphology in Labeled Polymer Blends with MAS NMR
}

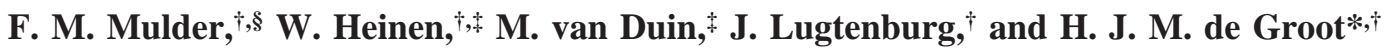 \\ Contribution from the Leiden Institute of Chemistry, Gorlaeus Laboratory, Leiden University, \\ P.O. Box 9502, 2300 RA Leiden, The Netherlands, and DSM Research, P.O. Box 18, \\ 6160 MD Geleen, The Netherlands
}

Received March 20, 1998. Revised Manuscript Received September 29, 1998

\begin{abstract}
A pulse sequence has been implemented for the determination of domain sizes in ${ }^{13} \mathrm{C}$ enriched organic materials with two-dimensional high-resolution magic angle spinning (MAS) NMR correlation spectroscopy. The method integrates the high resolution of ${ }^{13} \mathrm{C}$ MAS for selection and detection with an efficient polarization transfer across domain boundaries via strong ${ }^{1} \mathrm{H}$ dipolar interactions. A one-dimensional version with spectral editing allows for improved efficiency with respect to measurement time. With this MAS NMR and labeling approach heterogeneity at length scales from 1 to $100 \mathrm{~nm}$ can be determined for systems without long-range order. As an example, proton spin diffusion times up to $500 \mathrm{~ms}$ were used to study the morphology of a phase-separated semi-interpenetrating network of poly(styrene-co-acrylonitrile) and cross-linked poly(styrene-co-maleic anhydride). From the spin diffusion behavior a characteristic domain diameter $d=63 \pm 4 \mathrm{~nm}$ was calculated.
\end{abstract}

\section{Introduction}

Variation of the physical and chemical microstructure in polymer blends can have distinguishable effects on the properties of their macroscopic materials. In general, imaging of the full microstructure of polymer blends is feasible with microscopic techniques such as scanning or transmission electron microscopy or diffraction techniques such as X-ray. However, an experimental determination and characterization of phase separation and the resulting morphology at length scales shorter than $\approx$ $50 \mathrm{~nm}$ in amorphous polymers and other noncrystalline compounds is difficult. In addition, the resolution of small-angle $\mathrm{X}$-ray diffraction is often insufficient because of a low contrast. Recently, ${ }^{1} \mathrm{H}$ solid-state NMR methods have been developed for determining essential characteristics of the morphology of blends and compatibalizers. ${ }^{1-5}$ These spin diffusion methods determine the time-dependent transfer of nuclear polarization between different nuclei under influence of nuclear dipolar couplings. From this, distance information between the different nuclei is obtained with use of a spin diffusion coefficient. By observing the spin diffusion via strong homonuclear dipolar interactions, Spiess and co-workers have succeeded in determining the characteristic domain sizes and have estimated characteristics of their shape. ${ }^{6}$ However, these NMR methods require a considerable dispersion of ${ }^{1} \mathrm{H}$ signals to allow the selection

\footnotetext{
Leiden University.

$\doteqdot$ DSM Research.

$\S$ Present address: Interfaculty Reactor Institute, Delft University of Technology, Mekelweg 15, 2629 JB Delft, The Netherlands.

(1) Caravatti, P.; Neuenschwander, P.; Ernst R. R. Macromolecules 1985, $18,119$.

(2) Caravatti, P.; Neuenschwander, P.; Ernst, R. R. Macromolecules 1986, $19,1889$.

(3) Claus, J.; Schmidt-Rohr, K.; Spiess, H. W. Acta Polym. 1993, 44, 1.

(4) Veeman, W. S. In NMR of Polymer Composites and Blends; Maciel G., Ed.; NATO ASI, 1994.
}

(5) Schaefer, J.; Klug, C. A.; Zhu, W. Macromolecules 1997, 30, 1734.

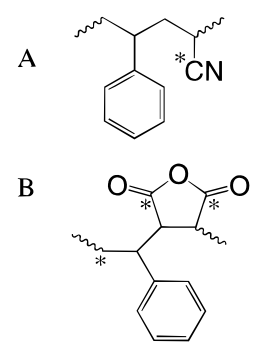

Figure 1. Chemical structure of poly(styrene-co-acrylonitrile) (A) and poly(styrene-co-maleic anhydride) (B). The asterisks indicate the positions of the labels.

of resonances from one polymer component based on the ${ }^{1} \mathrm{H}$ chemical shifts. By consequence, they can only be applied to a restricted subset of materials.

The specific aim of this study is to demonstrate that heteronuclear solid state NMR in conjunction with site-selective isotope labeling can provide a crucial enhancement of the resolution when specifically labeled polymers are used, engineered with isotopes at strategic positions in every monomeric unit. This is illustrated for a phase-separated poly(styrene-co$\left[{ }^{13} \mathrm{CN}\right]$ acrylonitrile) (SAN) and poly $\left(\left[\beta-{ }^{13} \mathrm{CH}_{2}\right]\right.$ styrene-co$\left[{ }^{13} \mathrm{CO}\right]$ maleic anhydride) (SMA) copolymer blend (Figure 1). The ${ }^{1} \mathrm{H}$ NMR responses of the two copolymers strongly overlap. It will be shown how the heterogeneity of the blend can be examined by analyzing the ${ }^{1} \mathrm{H}$ spin diffusion behavior, with shift selection and detection in the ${ }^{13} \mathrm{C}$ domain, using the isotope labels. Two pulse sequences are presented, one for $2-\mathrm{D}{ }^{13} \mathrm{C}-$ ${ }^{13} \mathrm{C}$ correlation spectroscopy and a $1-\mathrm{D}$ experiment where the equilibration of polarization originating from protons in the vicinity of a labeled site is probed in a simple inversion recovery

(6) Schmidt-Rohr, K.; Spiess, H. W. Multidimensional Solid-State NMR and Polymers; Academic Press: London, 1994. 
experiment. In both schemes magnetization is transferred back and forth between ${ }^{1} \mathrm{H}$ and ${ }^{13} \mathrm{C}$ by using three straightforward heteronuclear Hartmann-Hahn matching periods. In this way, efficient ${ }^{1} \mathrm{H}$ spin diffusion is integrated with high-resolution ${ }^{13} \mathrm{C}$ selection and detection to provide an effective method for the characterization of morphology and phase separation from the molecular level up to $10^{2} \mathrm{~nm}$ in labeled polymer blends.

\section{Materials and Methods}

The labeled SAN and SMA copolymers were obtained by total synthesis. $\left[1,4-{ }^{13} \mathrm{C}_{2}\right]$ Maleic anhydride (MA) was prepared from $\left[1-{ }^{13} \mathrm{C}\right]-$ acetic acid according to the methods described earlier. ${ }^{7}\left[1-^{13} \mathrm{C}\right]-$ Acrylonitrile (AN) was synthesized starting from $30 \mathrm{~g}$ of $\mathrm{K}^{13} \mathrm{CN}$, employing a flash vacuum thermolysis as the key step. ${ }^{8}$ For the synthesis of $\left[\beta-{ }^{13} \mathrm{C}\right]$ styrene, iodo $\left[{ }^{13} \mathrm{C}\right]$ methane was first reacted with triphenylphosphine to give $\left[{ }^{13} \mathrm{C}\right]$ methyltriphenylphosphonium iodide. This Wittig salt was deprotonated with sodium hydride and subsequently reacted with benzaldehyde, yielding $\left[\beta-{ }^{13} \mathrm{C}\right]$ styrene. Incorporation of the labels was confirmed with ${ }^{13} \mathrm{C},{ }^{1} \mathrm{H}$ NMR and MASS spectrometry.

Poly $\left(\left[\beta-{ }^{13} \mathrm{C}\right]\right.$ styrene-co- $\left[1,4-{ }^{13} \mathrm{C}_{2}\right]$ maleic anhydride $)$ with 27 wt $\%$ $\left[1,4-{ }^{13} \mathrm{C}_{2}\right]$ MA (MW $4 \times 10^{4} \mathrm{~g} / \mathrm{mol}$ ) was prepared by using an optimized starved feed procedure. ${ }^{9}$ The MA content was determined, using a calibrated infrared spectroscopic method, from the ratio of the intensities of the $\mathrm{C}=\mathrm{O}$ stretch absorption at $1807 \mathrm{~cm}^{-1}$ and the $\mathrm{CH}_{2}$ bend absorption at $1493 \mathrm{~cm}^{-1}$.

For the preparation of poly(styrene- $\mathrm{co}-\left[1-{ }^{13} \mathrm{C}\right]$ acrylonitrile) with 27 wt $\%$ of $\left[1-{ }^{13} \mathrm{C}\right]$ acrylonitrile $\left(\left[{ }^{13} \mathrm{CN}\right] \mathrm{SAN} 27\right), 4.80 \mathrm{~g}$ of freshly distilled styrene, $2.70 \mathrm{~g}$ of $\left[1-{ }^{13} \mathrm{C}\right]$ acrylonitrile, $1.0 \mathrm{~g}$ of a $5 \mathrm{wt} \%$ solution of sodium stearate in water, $60 \mathrm{mg}$ of tert-dodecylmercaptane, and 10 $\mathrm{mg}$ of sodium persulfate were added to $45.0 \mathrm{~g}$ of water. The emulsion was stirred at $70{ }^{\circ} \mathrm{C}$ for $2 \mathrm{~h}$ and coagulated in a 5-fold excess of methanol, which was acidified with 1 vol $\%$ of saturated aqueous hydrochloric acid. The product was washed thoroughly with methanol and dried for $24 \mathrm{~h}$ at $70{ }^{\circ} \mathrm{C}$ at reduced pressure. With use of elemental $\mathrm{C}, \mathrm{H}$, and $\mathrm{N}$ analysis a $27.0 \mathrm{wt} \%\left[1{ }^{13} \mathrm{C}\right]$ acrylonitrile content was found.

Both copolymers are well below their glass temperatures at room temperature: The SMA has a $T_{g} \approx 162{ }^{\circ} \mathrm{C}$ and the SAN has a $T_{g} \approx$ $112{ }^{\circ} \mathrm{C}$. To blend $\left[{ }^{13} \mathrm{CN}\right] \mathrm{SAN} 27$ and $\left[{ }^{13} \mathrm{CH}_{2},{ }^{13} \mathrm{CO}\right] \mathrm{SMA} 27$, the copolymers were codissolved in acetone. After evaporation of the solvent the blend was dried at $50{ }^{\circ} \mathrm{C}$ at reduced pressure. A semi-IPN with domains of SAN in a continuous network of SMA was created by crosslinking SMA with 4,4'-methylenedianiline in the solution of acetone.

CP/MAS NMR experiments were performed at room temperature with a Bruker MSL 400 spectrometer operating at a ${ }^{13} \mathrm{C}$ frequency of $100.6 \mathrm{MHz}$, using a $4 \mathrm{~mm}$ double resonance MAS probe with infrared spinning speed detection. During all experiments the spinning speed was kept stable within $\pm 3 \mathrm{~Hz}$. Continuous wave decoupling with nutation frequencies around $80 \mathrm{kHz}$ in the proton channel were used. ${ }^{13} \mathrm{C}$ shifts are externally referenced with respect to the $\left[1-{ }^{13} \mathrm{C}\right]$ response of labeled glycine at $176.04 \mathrm{ppm}$ downfield from TMS.

\section{Results and Discussion}

The rate of magnetization transfer $P(t)$ between two nuclei $j$ and $k$ is given by ${ }^{10}$

$$
P(t)={ }^{1} / 2 \pi g_{0}{ }^{j k}\left(\omega_{j}-\omega_{k}\right) \omega_{\mathrm{D}}{ }^{2} t
$$

with $g_{0}{ }^{j k}\left(\omega_{j}-\omega_{k}\right)$ proportional to the overlap between the NMR responses of $j$ and $k$, while $\omega_{\mathrm{D}}$ measures the dipolar coupling

(7) Heinen, W.; Rosenmöller, C.; Wenzel, C. B.; de Groot, H. J. M.; Lugtenburg, J.; van Duin, M. Macromolecules 1996, 29, 1151.

(8) van den Berg, E. M. M.; Richardson, E. E.; Lugtenburg, J.; Jenneskens, L. W. Synth. Commun. 1987, 17, 1189.

(9) Heinen, W.; Wenzel, C. B.; Rosenmöller, C.; Mulder, F. M.; Boender, G. J.; Lugtenburg, J.; de Groot, H. J. M.; van Duin, M.; Klumperman, B. Macromolecules 1998, 31, 7404. Heinen, W. Ph.D. Thesis, Leiden University, 1996.

(10) Abragam, A. The Principles of Nuclear Magnetism; Oxford University Press: London, 1961.
A

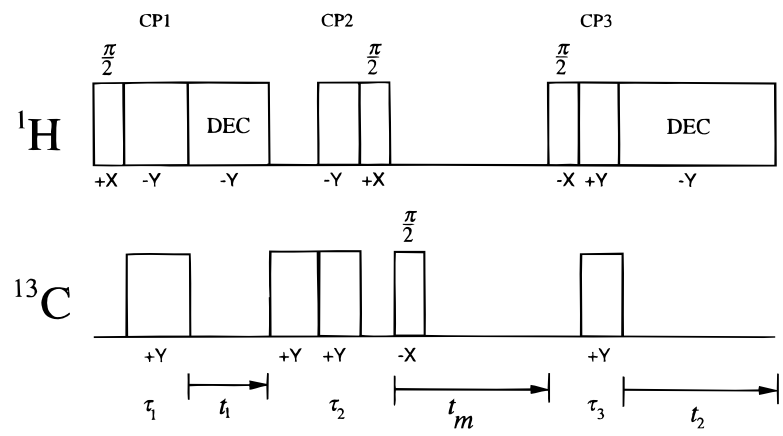

$\mathrm{TPPI}=0, \pi / 2, \pi, 3 \pi / 2$

B

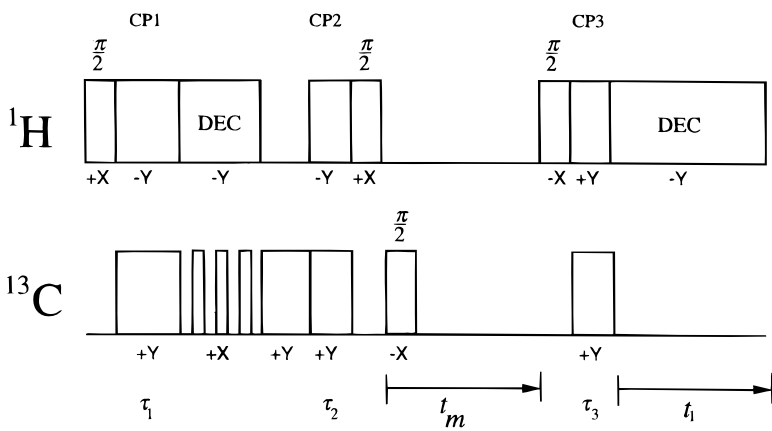

Figure 2. Pulse sequences for probing ${ }^{1} \mathrm{H}$ spin diffusion with ${ }^{13} \mathrm{C}$ selection and detection, using $2-\mathrm{D}{ }^{13} \mathrm{C}-{ }^{13} \mathrm{C}$ correlation spectroscopy (A) and the 1-D inversion recovery experiment including a selective rotor-synchronized DANTE inversion period (B).

Table 1. Phases for the $\mathrm{CP}^{3}$ Pulse Sequence ${ }^{a}$

\begin{tabular}{cccccc}
\hline scan & $\phi_{1}$ & $\phi_{2}$ & $\phi_{3}$ & $\phi_{4}$ & $\phi_{\text {rec }}$ \\
\hline 1 & $+X$ & $-Y$ & $-X$ & $+Y$ & $-X$ \\
2 & $+X$ & $+Y$ & $-X$ & $+Y$ & $-X$ \\
3 & $-X$ & $-Y$ & $+X$ & $-Y$ & $+X$ \\
4 & $-X$ & $+Y$ & $+X$ & $-Y$ & $+X$ \\
\hline
\end{tabular}

${ }^{a}$ A full cycle is 4 scans.

between the two nuclei. Spin diffusion involves the relayed transfer of magnetization through dipolar couplings. In polymers below their glass temperature, ${ }^{13} \mathrm{C}$ spin diffusion is slow compared to ${ }^{1} \mathrm{H}$ spin diffusion. For ${ }^{13} \mathrm{C}$ the dipolar couplings are weak, $\omega_{\mathrm{D}}<100 \mathrm{~Hz}$, the density of nuclei is low, and the overlap of NMR lines is small due to the large shift dispersion. This effectively prohibits spin diffusion over distances larger than $1 \mathrm{~nm}$. On the other hand, the ${ }^{1} \mathrm{H}$ spins are abundant, the dipolar couplings are strong $\left(\omega_{\mathrm{D}}>20 \mathrm{kHz}\right)$, and the lines strongly overlap. However, since the chemical shift dispersion is generally small, the use of the intrinsically large dispersion of the resonances for high-resolution selection and detection in general will be indispensable. To provide sufficient range for morphology characterization in phase-separated blends, ${ }^{13} \mathrm{C}$ isotope labeling is a prerequisite.

Two pulse programs combining MAS ${ }^{13} \mathrm{C}$ detection and mixing by efficient ${ }^{1} \mathrm{H}$ spin diffusion were implemented. Figure $2 \mathrm{~A}$ and Table 1 show the sequence for $2 \mathrm{D}$ homonuclear correlation spectroscopy. With a $\pi / 2{ }^{1} \mathrm{H}$ pulse with phase $X$ followed by a Hartmann-Hahn contact period (CP1), transverse ${ }^{13} \mathrm{C}$ coherence is established. After the evolution during $t_{1}$ the remaining coherence in the $X Y$ plane is suppressed by $T_{2}$ dephasing processes with a short purge period, while the ${ }^{13} \mathrm{C}$ magnetization along $\pm X$ is maintained with a spin-lock pulse. Subsequently, the power is turned on again, with phase $-Y$, 

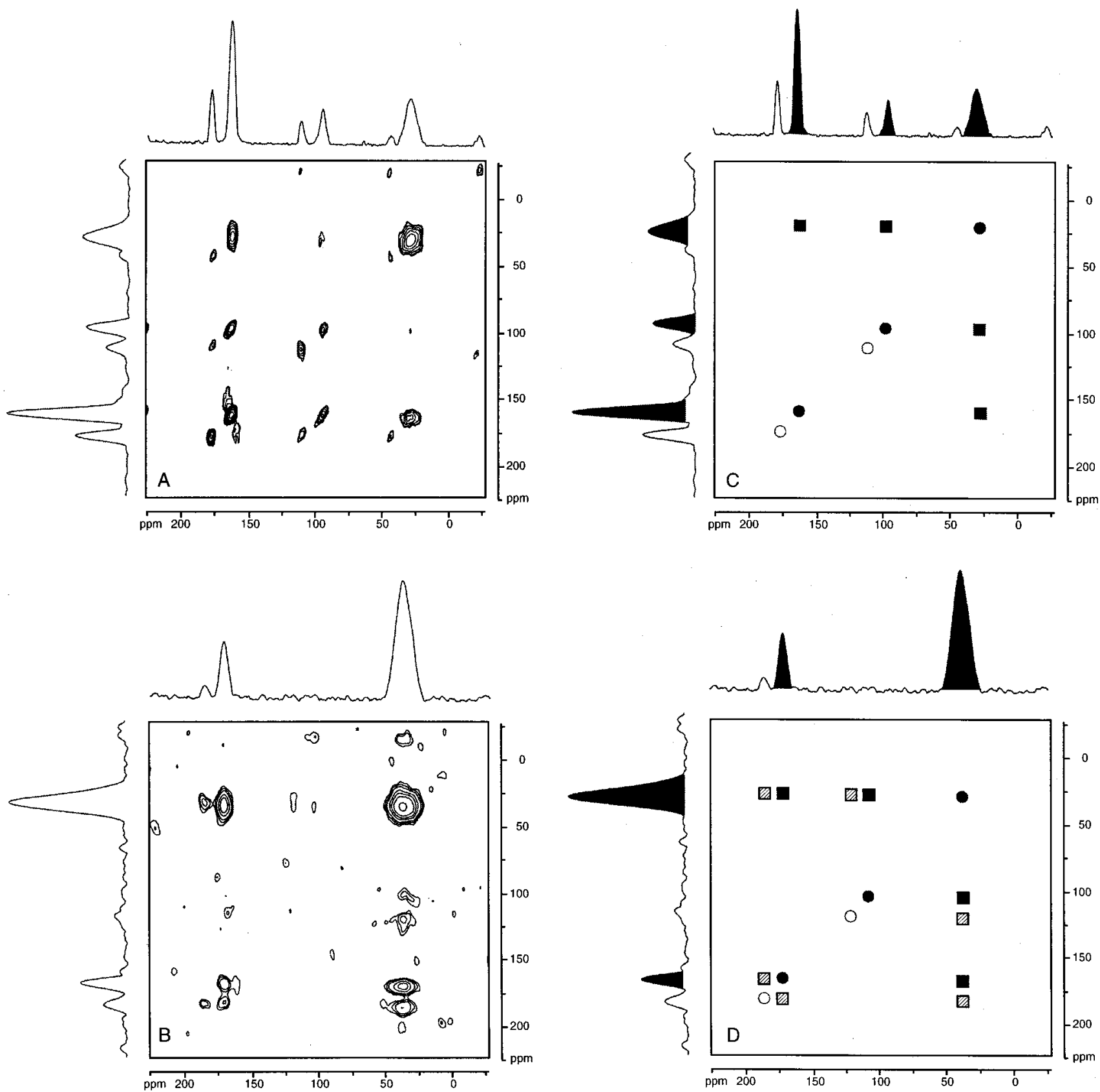

Figure 3. 2-D ${ }^{13} \mathrm{C}-{ }^{13} \mathrm{C}$ correlation spectra of the phase-separated semi-interpenetrating network of selectively labeled SAN and $70 \%$ cross-linked SMA. The left part of the figure shows the experimental data with durations $\tau_{1}, \tau_{2}$, and $\tau_{3}$ of 2 , 1 , and 1 ms, respectively. The spin-lock time following $t_{1}$ was $400 \mu \mathrm{s}$, and a spinning speed $\omega_{\mathrm{r}} / 2 \pi=6550 \pm 3 \mathrm{~Hz}$ was used. (A) Dataset collected with a mixing time $t_{m}=0 \mathrm{~ms}$. (B) Experimental data for $t_{m}=100 \mathrm{~ms}$. The ${ }^{13} \mathrm{C}$ peak in SAN has an isotropic chemical shift of $114 \mathrm{ppm}$, with a strong sideband at $180.5 \mathrm{ppm}$ that yields the more intense interdomain correlations. The ${ }^{13} \mathrm{CO}$ and ${ }^{13} \mathrm{CH}_{2}$ of SMA resonate with 196 and $20 \mathrm{ppm}$, respectively. The right part schematically depicts the intradomain and interdomain transfer events $(\mathrm{C}, \mathrm{D})$. The black circles correspond to the SMA labels and the open circles to the centerband and sideband of the SAN label. The black squares result from intradomain magnetization transfer, between the two different SMA labels. The gray squares in the bottom right schematic representation (D), for long $t_{m}$, indicate the cross-peaks that can be expected due to ${ }^{1} \mathrm{H}$ spin diffusion between the SAN and SMA labels across the phase boundaries. Several of these were observed experimentally, in the bottom left panel (B).

providing another heteronuclear matching period to transfer the ${ }^{13} \mathrm{C}$ magnetization back to the protons (CP2). A $\pi / 2{ }^{1} \mathrm{H}$ pulse with phase $X$ is used to flip the proton magnetization back to the $\pm z$-axis and spin diffusion can proceed during $t_{m}$. After the mixing period a $\pi / 2$ pulse with phase $-X$ flips the polarization back again to the $X Y$ plane, and a third crosspolarization period (CP3) is applied for ${ }^{13} \mathrm{C}$ detection. Phase cycling of the spin-lock pulses during $\mathrm{CP} 2$ compensates for ${ }^{1} \mathrm{H}$ $T_{1}$ relaxation effects. The ${ }^{13} \mathrm{C}$ magnetization remaining in the $X Y$ plane after $\mathrm{CP} 2$ is transferred to the $z$-axis and eliminated also by the cycling of the receiver phase. For this reason only proton spin diffusion is measured by the pulse sequence. A time proportional phase increment (TPPI) is included in the CP1 period. Since three matching periods are characteristic for the sequence, we use the acronym ' $\mathrm{CP}^{3}$ ',

In Figure 3A,B two spectra are shown with $t_{m}=0$ and 100 $\mathrm{ms}$, respectively. The spinning speed was $6650 \pm 3 \mathrm{~Hz}$. The three CP times were $\tau_{1}=2 \mathrm{~ms}, \tau_{2}=1 \mathrm{~ms}$, and $\tau_{3}=1 \mathrm{~ms}$. The ${ }^{1} \mathrm{H}$ dephasing period prior to the $\mathrm{CP} 2$ was $400 \mu \mathrm{s}$, which was sufficient to suppress the remaining ${ }^{1} \mathrm{H}$ transverse magnetization beyond detection. The isotropic shift of the ${ }^{13} \mathrm{CN}$ label in SAN is $114 \mathrm{ppm}$, and the ${ }^{13} \mathrm{CO}$ and ${ }^{13} \mathrm{CH}_{2}$ labels in SMA have shifts 
of 196 and $20 \mathrm{ppm}$, respectively. With the relatively slow spinning used for the experiments in Figure 3, the first downfield MAS sideband of the $\mathrm{SAN}{ }^{13} \mathrm{C}$ label at $180.5 \mathrm{ppm}$ is considerably stronger than its centerband at $114 \mathrm{ppm}$. This reflects the large and almost uniaxial shift anisotropy of the nitrile ${ }^{13} \mathrm{C}$. After mixing times of typically $40-100 \mathrm{~ms}$, several cross-peaks between the ${ }^{13} \mathrm{CN}$ group in $\mathrm{SAN}$ and the ${ }^{13} \mathrm{CH}_{2}$ and ${ }^{13} \mathrm{CO}$ groups in SMA are detected, revealing spin diffusion between the SAN and SMA domains. As noted before, direct ${ }^{13} \mathrm{C}$ spin diffusion between SAN and SMA is almost impossible at this spinning speed due to a lack of overlap between SAN and SMA ${ }^{13} \mathrm{C}$ NMR lines. In addition, it was suppressed by the phase cycling filter that was used for the collection of the data (Table $1)$.

From the $t_{m}=0 \mathrm{~ms}$ spectrum in Figure $3 \mathrm{~A}$ it can be concluded that the total Hartmann-Hahn matching time $\left(\tau_{1}+\right.$ $\tau_{2}=2 \mathrm{~ms}$ ) after the evolution period $t_{1}$ is sufficient to give strong intramolecular cross-peaks between the ${ }^{13} \mathrm{CO}$ and ${ }^{13} \mathrm{CH}_{2}$ within SMA. This implies that homonuclear magnetization transfer between protons during $\mathrm{CP}$ easily covers the short average distance of $3.0 \pm 0.1 \AA$ between these two labels within SMA (Figure 1A). A schematic representation of the intradomain transfer dataset is shown in the top right panel.

The 2D version of the spin diffusion experiment shows unambiguously that intermolecular proton polarization transfer occurs across domain interfaces during $t_{m}$ and can be detected with ${ }^{13} \mathrm{C}$ NMR. A $1 \mathrm{D}$ version of the program was also implemented, which is more efficient with respect to overall measurement time than the 2D version (Figure 2B). A rotorsynchronized DANTE pulse sequence with phase $+X$ is used to reverse selectively the magnetization associated with the ${ }^{13} \mathrm{CN}$ labels of SAN before the spin-lock is applied. ${ }^{11,12}$ With a rotorsynchronized DANTE sequence, the spacing between the pulses is equal to the inverse of a rotor period, to reverse the entire MAS pattern. With 3 pulses the cumulative $180^{\circ}$ pulse takes $0.45 \mathrm{~ms}$, yielding a selectivity of $\approx 1 \mathrm{kHz}$. This is adequate since it corresponds to the line width of the ${ }^{13} \mathrm{CN}$ label.

The intensity $I_{\mathrm{CN}}$ of the ${ }^{13} \mathrm{CN}$ MAS response of SAN can be normalized relative to the saturation value $I_{\mathrm{CN}}^{\mathrm{sat}}$, and $I_{\mathrm{CN}}$ can also be corrected for the $T_{1}$ relaxation. This yields

$$
\hat{I}_{\mathrm{CN}}=\frac{I_{\mathrm{CN}} \exp \left(\frac{t_{m}}{T_{1}}\right)+I_{\mathrm{CN}}^{\mathrm{sat}}}{2 I_{\mathrm{CN}}^{\mathrm{sat}}}
$$

In Figure 4 the normalized intensity $\hat{I}_{\mathrm{CN}}$ of the $\mathrm{CN}$ peak is plotted versus the square root of the mixing time $t_{m}{ }^{1 / 2}$. The zero in the vertical scale indicates complete reversal of the signal, while 1 corresponds to full relaxation to the equilibrium. Every data point in Figure 4 represents $4 \mathrm{~h}$ of measurement time (8000 scans). There is considerable polarization transfer already for $t_{m}=0 \mathrm{~ms}$ (total inversion of the ${ }^{13} \mathrm{CN}$ signal). Probably spin diffusion from SAN to SMA occurs during the last two heteronuclear Hartmann-Hahn periods in the interphase, which is the most intimately mixed fraction of the polymers. Such an interphase of a heterogeneous polymer blend has been observed with other NMR techniques. ${ }^{13}$

(11) Bodenhausen, G.; Freeman, R.; Morris, G. A. J. Magn. Reson. 1976, 23,171 .

(12) Morris, G. A.; Freeman, R. J. Magn. Reson. 1978, 29, 433.

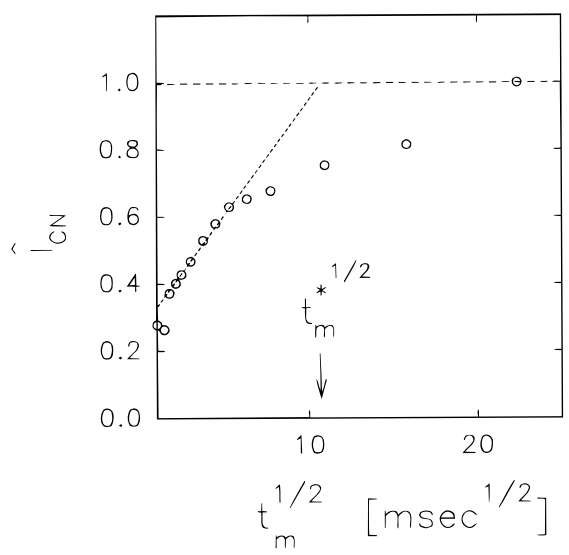

Figure 4. The intensity of the $\mathrm{CN}$ sideband pattern of ${ }^{13} \mathrm{C}$ labeled SAN versus the square root of the spin diffusion period $t_{m}$. As described in the text, the point at $t_{m}=t_{m}{ }^{*}$ at which the dashed lines cross determines the characteristic phase separation domain size $d$.

In the model for spin diffusion analysis described in ref 6 , the characteristic domain size $d$ is defined as the diameter of a spherical domain of either type of the two polymers in the blend. In the analysis, the line with the slope of the first points in Figure 4 crosses the saturation level 1.0, in the point defined as $t_{m}=$ $t_{m} *$. The expression

$$
d=6\left(4 D t_{m}^{*} / \pi\right)^{1 / 2}
$$

yields a characteristic domain size of $d=63 \pm 4 \mathrm{~nm}$ when the spin diffusion coefficient $D=0.7 \pm 0.1 \mathrm{~nm} \mathrm{~ms}^{-1}$ is used. This $D$ is considered a normal value for these materials. ${ }^{6}$ Hence, heterogeneity at length scales from 1 to $100 \mathrm{~nm}$ can be characterized inside isotopically enriched organic compounds without long-range order. A detailed study of labeled SAN and SMA blends prepared with varying cross-link densities is in preparation.

\section{Conclusions}

With the $\mathrm{CP}^{3}$ pulse sequence, high-resolution ${ }^{13} \mathrm{C}$ correlation spectra can be obtained with proton spin diffusion. After selective enrichment with isotopes, structural features in organic compounds without long-range order can be probed. Adequate signal-to-noise ratios were obtained within several hours of measurement time. It is possible to determine average distances between selectively enriched atoms or between entire groups of atoms. We report transfer of polarization across domain barriers, which is potentially useful for the study of, e.g., pattern labeled biological complexes. Under favorable conditions, uniformly enriched material also may be used, which would simplify the preparation of samples considerably.

Acknowledgment. We wish to thank C. Erkelens for technical support. This work is part of the research program of the "Stichting Technische Wetenschappen" (STW, Contract No. 8-251), which is financially supported by the "Nederlandse Organisatie voor Wetenschappelijk Onderzoek" (NWO). H.J.M.d.G. is a recipient of a NWO-PIONIER research grant.

JA9809511

(13) Tong, G.; Pan, Y.; Afeworki, M.; Poliks, M. D.; Schaefer, J. Macromolecules 1995, 28, 1719. 